\title{
Efficacy of Liu-zi-jue in Patients with 2019 Novel Coronavirus Pneumonia (COVID-19): structured summary of a study protocol for a randomized controlled trial
}

\author{
Shuaipan Zhang ${ }^{1,2+}$, Zhizhen $\mathrm{Lv}^{1,2+}{ }^{+}$, Qingguang Zhu ${ }^{2 \dagger}$, Wuquan Sun ${ }^{1}$, Fei Yao ${ }^{3}$, Lei Fang ${ }^{3}$, Yanbin Cheng ${ }^{2}$, \\ Zhiwei $\mathrm{Wu}^{1,2}$ and Min Fang ${ }^{1,2,3^{*+}+}$
}

\begin{abstract}
Objectives: Efficacy of conventional treatment plus the complementary therapy Liu-zi-jue (a mind-body exercise) to treat patients with mild COVID-19.

Trial design: The study is a single-center 2 arm, randomized controlled trial with parallel-group design.
\end{abstract}

Keywords: COVID-19, Randomized controlled trial, protocol, Liu-zi-jue, Conventional therapy

\section{Participants}

The trial will recruit 186 hospital patients with mild and general symptoms of COVID-19 pneumonia from 19th February to 31st July 2020. Diagnosis will follow the criteria of the National Health Commission \& State Administration of Traditional Chinese Medicine Diagnostic and Treatment Protocol for Novel Coronavirus Pneumonia (The 7th Trial Version). Trial Inclusion criteria: (1) No restrictions on gender and age; (2) Patients diagnosed with mild pneumonia (slight clinical symptoms, no pneumonia manifestations on imaging), general pneumonia patients (with fever, respiratory tract symptoms, etc., imaging showed pneumonia but no multiple organ damage); (3) Hospitalized patients; (4) Volunteer to join the trial and sign the "informed consent; (5) Agree not to perform other exercise activities. Trial

\footnotetext{
*Correspondence: fangmin19650510@163.com

'Shuaipan Zhang, Zhizhen Lv, Qingguang Zhu and Min Fang contributed equally to this work.

'Tuina Department, Yue yang Hospital of Integrated Traditional Chinese and Western Medicine, Shanghai University of Traditional Chinese Medicine, Shanghai 200437, China

${ }^{2}$ Institute of Tuina, Shanghai Institute of Traditional Chinese Medicine, Shanghai 200437, China

Full list of author information is available at the end of the article
}

Exclusion criteria: (1) patients with severe diseases such as cardiovascular, cerebrovascular, hematopoietic, digestive system or mental illness; (2 )pregnant and lactating women; (3) respiratory frequency> 30 times $/ \mathrm{min}$, showing respiratory failure; (4) complicated with other organ failure requires treatment by respiratory intensive care unit (ICU); (5) those who do not want to join the trial. Participants will be recruited from inpatients at the Department of Infectious Diseases at the hospital in Hubei Province.

\section{Intervention and comparator}

Patients in the control group with mild pneumonia, will be treated according to the $7^{\text {th }}$ Trial Version)" and given symptomatic supportive treatment. The intervention group will be instructed in performing Liu-zi-jue exercise therapy twice a day until 3 months after discharge. Liu-zi-jue, a lowrisk traditional Chinese exercise, is widely used for the prevention and treatment of respiratory diseases as a complementary therapy. Its movements are relatively simple and are mainly composed of 6 groups of exercise combined with breathing and stretching, which is suitable for home practicing. There is no need to rely on expensive equipment and 
Liu-zi-jue can be practiced individually or in groups without the limitation of sports experience and age.

\section{Main outcomes}

The primary outcome includes the Length of stay, Modified Borg Dyspnea Score, Fatigue Scale-14 Score, Patient Health Questionnaire-9 Score, which will evaluate the patient's respiratory symptoms and physical and mental health. The outcomes will be assessed at five points including the baseline, before treatment on the sixth day of hospitalization, the discharge day, 1 month after discharge and 3 months after discharge.

\section{Randomization}

Patients will be recruited and randomly assigned to the conventional treatment group and the intervention group conventional treatment plus Liu-zi-jue group in a 1:1 ratio. Researchers not delivering the intervention will get a random sequence number which is automatically generated by a random number generator (IBM Corp., Armonk, NY, USA). These will be sequentially placed in an opaque envelope. The research coordinator will open these random allocation envelopes and assign participants accordingly.

\section{Blinding (masking)}

Participants and Liu-zi-jue trainers are unable to be blinded to group assignment due to the nature of this physical intervention. To reduce the risk of bias, evaluators, data managers, and statisticians will be unaware of group assignments in the outcome evaluation process and data analysis.

\section{Numbers to be randomized (sample size)}

A total of 186 patients will be randomize 93 participants into the intervention group and 93 into the conventional treatment group.

\section{Trial Status}

This trial is still recruiting patients now. The first patient was recruited on February 19 and is scheduled to complete recruitment on May 30. The protocol is Version 2, March 26.

\section{Trial registration}

This trial was registered retrospectively in the Chinese Clinical Trial Registry on 26 April 2020. The registration number is ChiCTR2000032367.

\section{Full protocol}

We declare that the full protocol is attached as an additional file, accessible from the Trials website (Additional file 1). In the interest in expediting dissemination of this material, the familiar formatting has been eliminated; this Letter serves as a summary of the key elements of the full protocol. The study protocol has been reported in accordance with the Standard Protocol Items: Recommendations for Clinical Interventional Trials (SPIRIT) guidelines (Additional file 2).

\section{Supplementary information}

Supplementary information accompanies this paper at https://doi.org/10. 1186/s13063-020-04383-2.

Additional file 1. Full protocol.

Additional file 2. SPIRIT 2013 Checklist.

Additional file 3. Proof of Ethics.

Additional file 4. Funding proof.

\section{Acknowledgements}

Not applicable

Authors' contributions

SZ planned the study protocol and drafted the manuscript. ZL planned the study protocol and participated in the critical revision of the manuscript. QZ, carried out the Liu-zi-jue movements for patients and participated in the design of the protocol. MF managed the study and reviewed the manuscript. WS, FY, LF participated in designing the trial and helped to prepare the manuscript. ZW participated in the remote intervention supervision and revised the manuscript. All the authors have read and approved the final manuscript.

\section{Funding}

This study is supported financially by the project of Emergency scientific research project for prevention and control of new coronavirus (COVID-19) by Shanghai University of Traditional Chinese Medicine (first batch) (No fund number) and Shanghai's three-year action project on further accelerating the development of Traditional Chinese Medicine (ZY (2018-2020)-CCCX-2004-

02). The funding is widely used and applicable to this study. The funder had no role in the design of the study, analysis, collection, and interpretation of the data, or the writing and decision for publication of the manuscript.

Availability of data and materials Not applicable

Ethics approval and consent to participate

Ethics approval was requested and granted by the ethics committee of Yue yang Hospital of Integrated Traditional Chinese and Western Medicine (item number: 2020-002). Informed consent will be obtained from all study participants before starting any data collection by the clinical trial communicator. All participants will provide their consent in writing.

Consent for publication

Not applicable

Competing interests

The authors declare that they have no competing interests.

Author details

${ }^{1}$ Tuina Department, Yue yang Hospital of Integrated Traditional Chinese and Western Medicine, Shanghai University of Traditional Chinese Medicine,

Shanghai 200437, China. ${ }^{2}$ Institute of Tuina, Shanghai Institute of Traditional Chinese Medicine, Shanghai 200437, China. ${ }^{3}$ Shanghai University of

Traditional Chinese Medicine, Shanghai 201203, China.

Received: 4 May 2020 Accepted: 5 May 2020

Published online: 23 May 2020

\section{Publisher's Note}

Springer Nature remains neutral with regard to jurisdictional claims in published maps and institutional affiliations. 